\title{
Wir haben nichts zu verbergen, im Gegenteil!
}

\section{Christoph Bosshard}

Dr. med., Vizepräsident der FMH, Departementsverantwortlicher Daten, Demographie und Qualität

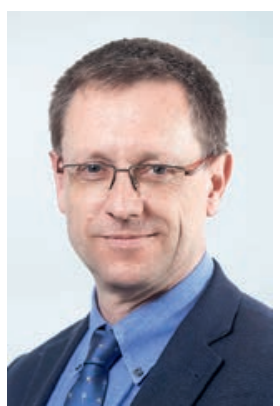

Nun ist es also wieder so weit: Ab dem 12. November beginnt die Datenerhebung des Bundesamtes für Statistik BFS betreffend ambulante Praxen und Zentren 2017. Sicher macht uns zusätzlicher Bürokram nicht wirklich Freude und wir fragen uns: weshalb? Was bringt es mir? Bringt es unseren Patientinnen und Patienten etwas? oder der Ärzteschaft?

Transparenz und eine verlässliche Datengrundlage sind die Basis für eine sachliche, faktenbasierte und lösungsorientierte Diskussion. Gemäss dem BAG und der BASS-Studie beläuft sich das reale Einkommen der Fachärztinnen und -ärzte in der Schweiz auf CHF 190577. In diesem Durchschnittswert ist jedoch ein Viertel der Ärzteschaft nicht berücksichtigt, nämlich die rund 10000 Assistenzärztinnen und -ärzte. Wie hoch der Anteil des Einkommens ist, welches durch die Prämiengelder der Schweizer Bürgerinnen und Bürger finanziert ist, wird aus dieser Studie ebenfalls nicht ersichtlich. Die Einkommenszahlen stammen aus dem Jahr 2014 vor den Tarifreduktionen durch den Bundesrat.

Mit der Erhebung MARS haben wir nun die Möglichkeit, die Erhebungen und Auswertungen deutlich differenzierter vorzunehmen. Es werden Umsätze getrennt

\section{Ab dem 12. November beginnt die Datenerhe- bung des Bundesamtes für Statistik BFS betref- fend ambulante Praxen und Zentren 2017.}

nach Grund- und Zusatzversicherung erhoben. Die Erträge, welche nichts mit der Praxistätigkeit zu tun haben, sind klar und transparent dargestellt. Somit ist nun durch die Publikation der MAS-2015-Resultate ${ }^{1}$ klar geworden, dass die bisher gemachten Aussagen der Ärzteschaft stimmen und die Kostensätze praxisambulant nicht «nur» $70 \%$, sondern sogar $72 \%$ betragen. Auch kann klar abgegrenzt werden, welche Einkünfte sozialversicherungsbasiert sind, denn lediglich diesbezüglich kann ein öffentliches Interesse betreffend Transparenz abgeleitet werden. Was kann uns also Besseres passieren, als dass unsere Aussagen vom Bund bestätigt und damit validiert werden?
Um die Datenqualität möglichst hoch zu halten und den administrativen Aufwand möglichst klein, bieten die Ärztekasse, NewIndex und die FMH jedem Mitglied wiederum an, seine dort vorhandenen Daten per Knopfdruck in den Fragebogen zu importieren. Das Bundesamt für Statistik setzt zusammen mit der FMH auf Überzeugungsarbeit und verzichtet auch weiterhin auf Sanktionen gegenüber denjenigen Mitgliedern, welche ihren gesetzlichen Pflichten nicht nachkommen wollen.

Was kann uns Besseres passieren, als dass unsere Aussagen vom Bund bestätigt und validiert werden?

Die Anonymisierung ist ebenfalls gewährleistet, dies auch im aufsichtsrechtlichen Teil. So legen die entsprechenden Grundlagen auf Verordnungs- und Reglementsebene fest, dass lediglich aggregierte Daten an die Datenempfänger (BAG, Versicherer, Kantone) weitergegeben werden dürfen, welche keine Rückschlüsse auf die Einzelpraxis ermöglichen.

Oft werde ich gefragt, was denn mit den Daten passiere, wenn sich die gesetzlichen Rahmenbedingungen ändern würden. Dazu ist klar zu sagen, dass sich gesetzliche Rahmenbedingungen ja nicht so einfach ohne Anlass ändern. Die konstruktive Zusammenarbeit mit dem BFS hilft uns hier. Weiter ist klar, dass Verwendungszwecke nicht nachträglich geändert werden dürfen, sondern erst proaktiv in Kraft treten können. Bei jeglicher Veränderung der juristischen Grundlagen erfolgen sofort Neuverhandlungen betreffend Zusammenarbeit. So ist gewährleistet, dass die FMH als aktiver Partner eingebunden bleibt.

Vor dem Hintergrund dieser Darlegungen empfiehlt der Zentralvorstand der FMH den Mitgliedern, an der nun startenden Datenerfassung des BFS teilzunehmen. Der Aufwand ist da, ein erheblicher Nutzen jedoch auch! Ich danke allen, welche uns hier unterstützen. Ebenfalls danke ich unseren Partnern und Mitarbeitenden für ihren wertvollen und unermüdlichen Einsatz! 